\title{
Regarding "Post-mortem CT lung findings at a medicolegal institute in SARS-CoV-2 RT-PCR positive cases with autopsy correlation"
}

\author{
Fabio De-Giorgio ${ }^{1,2} \odot$ Eva Bergamin ${ }^{2} \cdot$ Francesca Cittadini $^{1,2} \cdot$ Alessandro Cina $^{3} \cdot$ Giuseppe Vetrugno $^{1,2}$
}

Accepted: 18 September 2021 / Published online: 1 November 2021

(c) Springer Science+Business Media, LLC, part of Springer Nature 2021

With considerable interest, we read the article by O'Donnell et al. [1]. The article confirms our previous assumption [2] that the sole use of postmortem computed tomography (PMCT) should not be recommended as a tool for determining the cause of death of COVID-19 patients [1]. In this regard, we would like to share with the readers the case of a 42-year-old woman who died of complications of COVID-19 viral infection. We had the opportunity to examine the body by using two totalbody CT scans that were performed prior to the autopsy; the first exam was performed perimortem, whereas the second exam was performed at a PMI of $>72 \mathrm{~h}$. This allowed us to highlight the limitations of PMCT in terms of COVID-19 diagnosis.

From a clinical and forensic standpoint, COVID-19 represents an extremely complex issue. Patterns of groundglass opacity (GGO), multifocal consolidation, septal thickening, and pleural effusions have been reported in the few studies that focus on COVID-19-related PMCT findings [2, 3].

In our case, the first scan revealed a radiological picture suggestive of COVID-19 (Fig. 1A). Conversely, the second scan showed inconclusive findings, especially in terms of diagnostic purposes (Fig. 1B). The lung parenchyma appeared to be completely consolidated, due to the uniform spreading of postmortem changes; therefore, this was not indicative of a diagnosis. This is the reason why, for the second scan, we chose a mediastinal window over a pulmonary window (Fig. 1B); indeed, if we had used the latter method, we would have obtained an image of a "white lung".

We believe that if we consider the sole use of PMCT, this may be helpful in the perimortem period; however, it should not be recommended for the late postmortem diagnosis of COVID-19. This is mostly attributable to the fact that, with the onset of normal postmortem changes, the findings of the infection may be masked by the latter, as the two are similar. Conversely, the postmortem histological analysis of biological samples has been proven to be effective for the identification of the cause of death, regardless of the postmortem period $[4,5]$.

Thus, although our case represents only one case that describes these findings, our results were in accord with the authors' findings. They found non-specific PMCT evidence of COVID-19 pneumonia, as well as the absence of substantial findings in some COVID-19 positive cases. Moreover, in some cases, lung findings were concealed by normal postmortem alterations.

Fabio De-Giorgio

fabio.degiorgio@unicatt.it

1 Department of Healthcare Surveillance and Bioethics, Section of Legal Medicine, Università Cattolica del Sacro Cuore, Rome, Italy

2 Fondazione Policlinico Universitario IRCCS A. Gemelli, Rome, Italy

3 Department of Diagnostic Imaging, Oncological Radiotherapy and Hematology - Diagnostic Imaging Area, Fondazione Policlinico, Universitario Agostino Gemelli IRCCS, Università Cattolica del Sacro Cuore, Rome, Italy 


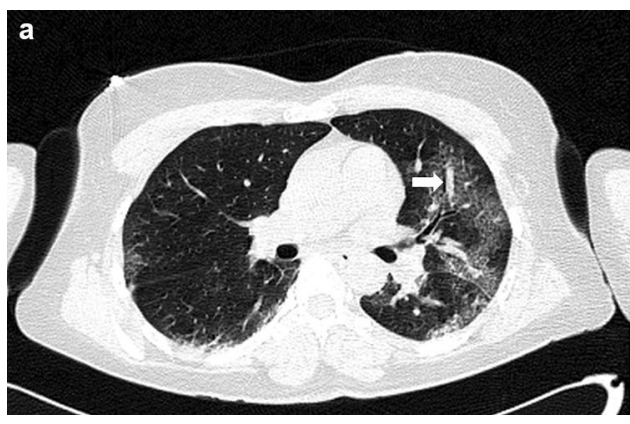

Fig. 1 A) Unenhanced CT scan (lung window) showing multiple bilateral peripheral nondependent "ground glass" opacities. In some areas enlarged pulmonary vessels are present (arrow) within opacities. CT findings are characteristic, even if not specific, of COVID-19 pneumonia. B) Postmortem CT (mediastinal window) acquired on the fourth day postmortem, before autopsy. Consolidation of parenchyma

\section{References}

1. O'Donnell C, Iles L, Woodford N. Post-mortem CT lung findings at a medicolegal institute in SARS-CoV-2 RT-PCR positive cases with autopsy correlation. Forensic Sci Med Pathol. 2021;23:1-10.

2. De-Giorgio F, Cittadini F, Cina A, Cavarretta E, Biondi-Zoccai G, Vetrugno $\mathrm{G}$, et al. Use of post-mortem chest computed tomography in Covid-19 pneumonia. Forensic Sci Int. 2021;325:110851.

3. Fitzek A, Sperhake J, Edler C, Schröder AS, Heinemann A, Heinrich F, et al. Evidence for systematic autopsies in COVID19 positive deceased: Case report of the first German investigated COVID-19 death. Rechtsmedizin (Berl). 2020;25:1-6.

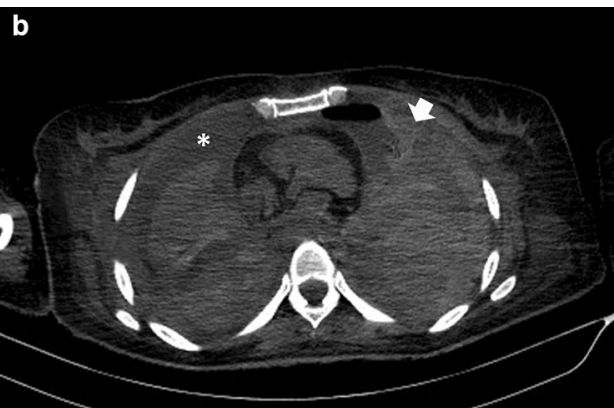

of both lungs is present. Bilateral pleural effusion is evident; with fluid density on the right (asterisk) and hematic density on the left side (arrow). This CT appearance with diffuse parenchymal involvement does not allow any evaluation of possible infectious disease of lungs

4. De-Giorgio F, Grassi VM, Bergamin E, Cina A, Del Nonno F, Colombo D, et al. Dying "from" or "with" COVID-19 during the pandemic: Medico-legal issues according to a population perspective. Int J Environ Res Public Health. 2021;18:8851.

5. De-Giorgio F, Vetrugno G. Is histological examination always necessary to determine a cause of death? Of course it is! Forensic Sci Med Pathol. 2014;10:477-8.

Publisher's Note Springer Nature remains neutral with regard to jurisdictional claims in published maps and institutional affiliations. 